\title{
A Finite Volume-Based High Order Cartesian Cut-Cell Method for Computational Aeroacoustics
}

\author{
Mihaela Popescu ${ }^{*}$ and Cheng-Feng Tai. ${ }^{\dagger}$ \\ Department of Mechanical and Aerospace Engineering \\ University of Florida, Gainesville, FL 32611 \\ Wei Shyy \\ Department of Aerospace Engineering \\ University of Michigan, Ann Arbor, MI 48109
}

\begin{abstract}
A finite volume-based high-order scheme with optimized dispersion and dissipation characteristics in cooperation with the Cartesian cut-cell technique is presented for aeroacoustics computations involving geometric complexities and nonlinearities. The field equation is solved based on an optimized prefactored compact finite volume (OPC-fv) scheme. The cut-cell approach handles the boundary shape by sub-dividing the computational cells in accordance with the local geometric characteristics and facilitates the use of numerical procedures with a desirable level of accuracy. The resulting technique is assessed by several test problems that demonstrate satisfactory performance.
\end{abstract}

\section{Nomenclature}

$c \quad=$ sound speed

$C F L=$ Courand-Friederichs-Lewy number $\rightarrow c \frac{\Delta t}{\min (\Delta x, \Delta y)}$

$D_{i}^{F}, D_{i}^{B}=$ forward and backward derivative operators in the point $i$

$\mathrm{fv} \quad=$ finite volume

$K^{(\mathrm{i})} \quad=$ computed in the stage $i$, in the Runge-Kutta time integration

$\mathrm{t}=$ time

$u_{i}^{B e}, u_{i}^{F e}=$ forward, and backward operator computed on east face in the cell $i$

$u_{i}^{B w}, u_{i}^{F w}=$ forward, and backward operator computed on west face in the cell $i$

$u_{i}^{e} \quad=$ the value of parameter $u$ on face $e$, in cell $i$

$u_{i}^{w} \quad=$ the value of parameter $u$ on face $w$, in cell $i$

$u^{(\mathrm{m})} \quad=$ the value of function $u$ in the stage $m$ in the Runge-Kutta time integration

$u^{n} \quad=$ the value of function $u$ in the $n$ iteration, it is related to time integration

$x, y=$ coordinate in space

$\Delta x, \Delta y, \Delta t=$ length of grid in space, in $x$ and $y$ direction, respectively time step size

$\omega \quad=$ frequency

\section{Introduction}

T $\mathrm{N}$ computational aero-acoustics (CAA), one needs to resolve dispersion and dissipation characteristics in order to 1 preserve form and amplitude of the wave(Hardin and Hussaini ${ }^{1}$, Tam and coworkers ${ }^{2,3}$ ). Furthermore, special care needs to be exercised in treating the boundaries to prevent the creation of spurious waves while still accounting for wave reflection and/or scattering. In order to meet these expectations, there are specific issues and challenges associated with the employment of a structured multiblock grid, an overlapping grid, or an unstructured grid ${ }^{4-9}$, other

\footnotetext{
* Ph.D. Student, Mechanical and Aerospace Engineering Department, mihaela@ufl.edu, Member AIAA

${ }^{\dagger}$ Ph.D. Student, Mechanical and Aerospace Engineering Department

^Clarence L. "Kelly” Johnson Collegiate Professor and Chair, Fellow AIAA, weishyy@umich.edu
} 
alternatives should also be pursued. In this work, we present an approach utilizing the Cartesian cut-cell approach based on the finite-volume - based scheme aimed at optimizing the dispersion and dissipation treatment. The Cartesian, cut-cell approach has been developed extensively for moving and complex boundary computations ${ }^{10-15}$. It uses a background Cartesian grid for the majority of the flow domain while creating sub-cells in the boundary regions to meet the geometric requirements. Special algorithms for complex geometries are constructed using flow properties and appropriate interpolation and extrapolation procedures. The low dispersion and dissipation scheme is based on an extension of the finite difference-based, optimized prefactored compact (OPC) scheme originally developed by Ashcroft and Zhang ${ }^{16}$ that we call OPC-fd. Recently, Popescu et al. ${ }^{17}$ have extended it using the finite volume concept that we call OPC-fv, creating a scheme to better satisfy the nonlinearity and conservation laws. The present technique, which consists of the optimized dispersion and dissipation characteristics and the cut-cell technique, offers desirable capabilities in both the interior and boundary regions. In addition to the detailed derivation of the numerical techniques, several test problems will be presented to highlight the performance characteristics of the proposed approach.

\section{Numerical schemes}

\section{A. Discretization in Space - The Finite Volume Optimized Prefactored Compact (OPC-fv) Scheme}

Consider the simple one-dimensional wave equation:

$$
\frac{\partial u}{\partial t}+c \frac{\partial u}{\partial x}=0
$$

To derive the discretized equation, we employ the grid point cluster shown in

Figure 1. We focus on the grid point $i$, which has the grid points $i-1$ and $i+1$ as its neighbors. The dashed lines define the control volume, and the letters $e$ and $w$ denote the east and west faces, respectively, of the control volume. For the one-dimensional problem under consideration, we assume a unit thickness in the $y$ and $z$ directions. Thus, we obtain

$$
\int_{e}^{w} \frac{\partial u}{\partial t} d x+c\left((A u)_{e}-(A u)_{w}\right)=0
$$

where $(\mathrm{A} u)_{e}$ and $(\mathrm{A} u)_{w}$ are the fluxes across the east and west faces, respectively.

Hence, the discretized wave Eq. (1) can be written as

$$
\frac{\partial \bar{u}}{\partial t} \Delta x+c\left((A u)_{e}-(A u)_{w}\right)=0
$$

where $\bar{u}$ is the averaged value of $u$ over a control volume.

Taking into account Eq. (3), we describe the general form of the approximation of $\frac{\partial u}{\partial x}$ in 1-D using the control volume concept:

$$
\frac{\partial u}{\partial x} \rightarrow \frac{1}{\Delta x}\left((u A)_{e}-(u A)_{w}\right)
$$
defined.

The finite volume scheme is determined by the way in which the value of the fluxes on the faces $e$ and $w$ are

For a better understanding of the OPC-fv form, we present $\mathrm{OPC}-\mathrm{fd}^{16}$. The factorized compact scheme in the finite difference approach is obtained by defining the forward and backward operators $D_{i}^{F}$ and $D_{i}^{B}$ such that 


$$
\left(\frac{\partial u}{\partial x}\right)_{i}=\frac{1}{2}\left(D_{i}^{B}+D_{i}^{F}\right)
$$

The generic stencils for the forward and backward derivative operators are given by

$$
\begin{gathered}
\eta_{F} D_{i+1}^{F}+\beta_{F} D_{i}^{F}=\frac{1}{\Delta x}\left[a_{F} u_{i+2}+b_{F} u_{i+1}+c_{F} u_{i}+d_{F} u_{i-1}+e_{F} u_{i-2}\right] \\
\eta_{B} D_{i}^{B}+\beta_{F} D_{i-1}^{B}=\frac{1}{\Delta x}\left[a_{B} u_{i+2}+b_{B} u_{i+1}+c_{B} u_{i}+d_{B} u_{i-1}+e_{B} u_{i-2}\right]
\end{gathered}
$$

The finite volume formulation of optimized prefactored scheme is obtained taking into account Eq.(4) that describes the approximation of the first order spatial derivative in the, (6) and (7), and the idea that the general form of approximation of the function in points $e$ and $w$ has similar forms (approximation of the function in the center of the cell - see

Figure 1). The value of the function in the center of the face is defined by the relations:

$$
\left\{\begin{array}{l}
u_{e}=0.5\left(u^{F e}+u^{B e}\right) \\
u_{w}=0.5\left(u^{F w}+u^{B w}\right)
\end{array}\right.
$$

where $u^{F e}, u^{B e}, u^{F w}$ and $u^{B w}$ are determined from:

$$
\begin{aligned}
& \eta u_{i+1}^{F e}+\beta u_{i}^{F e}=b u_{i+1}-d u_{i} \\
& \eta u_{i+1}^{F w}+\beta u_{i}^{F w}=b u_{i}-d u_{i-1} \\
& \eta u_{i}^{B e}+\beta u_{i-1}^{B e}=b u_{i}-d u_{i+1} \\
& \eta u_{i}^{B w}+\beta u_{i-1}^{B w}=b u_{i-1}-d u_{i}
\end{aligned}
$$

where the coefficients are the same as those in the OPC-fd scheme ${ }^{16}: \quad \eta=\eta^{\mathrm{F}}=\gamma^{B}, \quad \beta=\beta^{F}=\beta^{B}$, $b=b^{F}=-d^{B}, d=d^{F}=-b^{B}$

\section{B. Time Discretization - Low Dispersion and Low Dissipation Runge-Kutta Method}

$\mathrm{Hu}$ at al. ${ }^{18}$ considered time integration using the Runge-Kutta algorithm of the differential equation

$$
\frac{\partial u}{\partial t}=F(u)
$$

where the operator $F$ is function of $u$. An explicit p-stage algorithm advances the solution of Eq.(10) from the $n^{\text {th }}$ to the $(n+1)^{\text {th }}$ iteration: 


$$
\begin{aligned}
& u^{(0)}=u^{n} \\
& K^{(1)}=\Delta t F\left(u^{(0)}\right) \\
& \ldots \\
& K^{(i)}=\Delta t F\left(u^{(i-1)}\right) \\
& u^{(i)}=u^{n}+b_{i} K^{(i)} \quad i=1, \ldots, p \\
& \ldots \\
& u^{n+1}=u^{(p)}
\end{aligned}
$$

where $b_{p}=1$

The coefficients of the low dispersion and low dissipation Runge-Kutta (LDDRK) are obtained such that: i) the scheme has a certain order of accuracy; ii) the error of the amplification factor of the scheme over the specified phase range is minimized; iii) the amplification factor of the scheme is within one of the given stability limit.

In this work we use a two-step alternating scheme. In the first step we use four stages, and in the second we use six stages. The scheme is fourth-order accurate in time for a linear problem and second-order accurate for a nonlinear problem.

The specific procedure is given below.

1. four-stage

$$
\begin{aligned}
& K^{(1)}=\Delta t F\left(u^{n}\right) \\
& K^{(2)}=\Delta t F\left(u^{n}+\frac{1}{4} K^{(1)}\right) \\
& K^{(3)}=\Delta t F\left(u^{n}+\frac{1}{3} K^{(2)}\right) \\
& K^{(4)}=\Delta t F\left(u^{n}+\frac{1}{2} K^{(3)}\right) \\
& u^{n+1}=u^{n}+K^{(4)}
\end{aligned}
$$

2. six-stage

$$
\begin{aligned}
& K^{(1)}=\Delta t F\left(u^{n}\right) \\
& K^{(2)}=\Delta t F\left(u^{n}+0.17667 K^{(1)}\right) \\
& K^{(3)}=\Delta t F\left(u^{n}+0.38904 K^{(2)}\right) \\
& K^{(4)}=\Delta t F\left(u^{n}+\frac{1}{4} K^{(3)}\right) \\
& K^{(5)}=\Delta t F\left(u^{n}+\frac{1}{3} K^{(4)}\right) \\
& K^{(6)}=\Delta t F\left(u^{n}+\frac{1}{2} K^{(5)}\right) \\
& u^{n+1}=u^{n}+K^{(6)}
\end{aligned}
$$

In a later section, we will give an implementation example of the LDDRK scheme where we use the OPC-fv scheme for spatial discretization:

where

$$
F_{l}=-c\left(u_{l}^{e}-u_{l}{ }^{w}\right) / \Delta x
$$




$$
u_{l}^{e}=0.5\left(u_{l}^{B e}+u_{l}^{F e}\right) \text {, and } u_{l}^{w}=0.5\left(u_{l}^{B w}+u_{l}^{F w}\right)
$$

\section{The Cut-Cell Procedure}

The cut-cell method rearranges the computational cells in the vicinity of the interface via sub-division to conform to the specified boundary shape. Depending on the intersection between the grid line and the interface, the subdivided, or cut, cells can remain independent or can be merged into a neighboring cell in a given direction, e.g. a direction approximately normal to the solid face ${ }^{10-15}$. Accordingly, the interfacial cells are reorganized along with their neighboring cells to form new cells with triangular, trapezoidal, or pentagonal shapes.

The flux across the cell boundaries can be approximated by

$$
\oint f \cdot \vec{n} d s \approx \sum_{k=1}^{m} f_{k} n_{k}
$$

The flux on the face is computed based on the multi-dimensional interpolation method ${ }^{14,}{ }^{15}$. Since the OPC scheme considered here is fourth order, it is desirable to preserve the same order of accuracy around the boundary.

The Cartesian cut cell mesh approach follows the following steps:

1. Locate the intersection of the boundary using a Cartesian mesh.

2. Construct the background Cartesian mesh: The cells are flagged as solid cells, flow cells, or boundary cells. The boundary cells are those that either intersect the boundary or have a face in common with the boundary.

3. Determine the geometric characteristics of the boundary cells such as cell volume, the direction normal to the boundary, and other information.

4. Merge cells as necessary. A minimum acceptable cell area $S_{\min }$ is specified, and when the area of a cut cell is smaller than this value, it is merged into a neighboring cell. Determine the new characteristics of the merged cells.

For the trapezoid ACDE (see Figure 3), the finite volume approach can be used to approximate the wave equation:

$$
\int_{A B C D E}\left(\frac{\partial u}{\partial t}+\frac{\partial E}{\partial x}+\frac{\partial F}{\partial y}\right) d v=0
$$

where $d v$ is a volume element. Appling Stoke's theorem to Eq.(17), we have

$$
\frac{\partial u_{c}}{\partial t} S_{A B C D E}+\oint_{A B C D E}(E d y-F d x)
$$

The value of the integral can be approximated by

$$
\oint_{A B C D E}(E d y-F d x)=\int_{A C} E d y+\int_{C D}(E d y-F d x)+\int_{D E} E d y-\int_{E A} F d x
$$

The function $F$ and $E$ can be represented generically by the function $f$. The fluxes on the faces $\mathrm{AC}$ and $\mathrm{DE}$ can be approximate by

$$
\int_{A C} f d y=\int_{A B} f d y+\int_{B C} f d y
$$

An approximation of the value of the flux is given by:

$$
\int_{A C} f d y \approx f_{w}\left(y_{A}-y_{B}\right)+f_{s w}\left(y_{B}-y_{C}\right)
$$


The value of the flux at point $w$ is given by the specific formula for the boundary cell. The value of $f_{s w}$ is approximated using a polynomial that is fourth order in the $x$ and $y$ direction:

$$
f_{s w}=\sum_{i=0}^{4} \sum_{j=0}^{4} b_{i j} x^{i} y^{j}
$$

where the coefficients $b_{i j}$ are unknown. This interpolation has fourth-order accuracy in the evaluation of the flux on the cut cells. In this case, the value of the coefficients is obtained using the values of $f$ in 25 grid points. An example is given in Figure 3 where the value of the function is approximated using the following 25 points.

To solve $b_{i j}$, we use the following system of equations by expressing the function $f$ at 25 locations:

$$
\left\{\begin{array}{l}
f_{1} \\
f_{2} \\
\vdots \\
f_{25}
\end{array}\right\}=\left\{\begin{array}{llllll}
x_{1}^{4} y_{1}^{4} & x_{1}^{3} y_{1}^{4} & x_{1}^{4} y_{1}^{3} \cdots & x_{1} & y_{1} & 1 \\
x_{2}^{4} y_{2}^{4} & x_{2}^{3} y_{2}^{4} & x_{2}^{4} y_{2}^{3} \cdots & x_{2} & y_{2} & 1 \\
\cdots & \cdots & \cdots & \cdots & \cdots & \cdots \\
x_{25}^{4} y_{25}^{4} & x_{25}^{3} y_{25}^{4} & x_{25}^{4} y_{25}^{3} \cdots & x_{25} & y_{25} & 1
\end{array}\right\}\left\{\begin{array}{l}
b_{1} \\
b_{2} \\
\vdots \\
b_{25}
\end{array}\right\}
$$

The coefficients $b_{i j}$ from Eq.(22) become the coefficients $b_{1}, b_{2}, \ldots, b_{25}$ in Eq. (23). These coefficients can now be expressed in terms of values of $f$ at the twenty five points by inverting Eq.(23), i.e.

$$
b_{n}=\sum_{j=1}^{25} a_{n j} f_{j}, \quad n=1, \ldots 25
$$

where $a_{n j}$ are the elements of the inverse of the matrix in Eq.(23).

After $b_{n}$ is obtained, the value of $f$ at the center of $B C$ is expressed in the form

$$
f_{s w}=b_{1} x_{s w}^{4} y_{s w}^{4}+b_{2} x_{s w}^{3} y_{s w}^{4}+b_{3} x_{s w}^{4} y_{s w}^{3}+\cdots+b_{23} x_{s w}+b_{24} y_{s w}+b_{25}
$$

and using Eq.(24), the value of $f_{s w}$ can be rewritten as

$$
f_{s w}=\sum_{j=1}^{25} \alpha_{j} f_{j}
$$

Note that bi, $i=1,2, \ldots, 25$ are coefficients that depend only on the mesh, the location, and the orientation of the boundary. Therefore, with a fixed geometry, these coefficients can be computed once at the beginning of the solution procedure.

Now we turn to the calculation of the flux on the immersed face $C D$ of the cell $(i, j)$. To compute the flux on a solid boundary, we use the reflection boundary condition. In Figure $3 \mathrm{~b},\left(x_{i}, y_{j}\right)$ is the mass center of the boundary cell. We introduce a point across the boundary that is symmetrically opposite of the center of mass of face $C D$, and note it with $G$. The variable values in the ghost point $G$ are

$$
\left\{\begin{array}{l}
p_{G}=p_{i j} \\
\vec{u}_{G}=\vec{u}_{i j}-2\left(\vec{u}_{i j} \cdot \vec{n}\right) \vec{n}
\end{array}\right.
$$

where $\vec{n}$ is the normal vector of the solid boundary, the index $i j$ indicates the value of variable at point $\left(x_{i}, y_{j}\right)$, and the index $G$ indicates values at the ghost point $G$. The value of the flux on face $C D$ is approximate by 


$$
\left\{\begin{array}{l}
p_{C D}=\frac{p_{G}+p_{i j}}{2} \\
\vec{u}_{C D}=\frac{\vec{u}_{G}+\vec{u}_{i j}}{2}
\end{array}\right.
$$

Following simple algebra, we can observe that Eq.(28) assures that the velocity is zero in the normal direction, and the variation of pressure in the normal direction around the boundary is zero.

\section{Test Cases}

Three test cases have been selected to assess the performance of the presented approach: i) radiation from a baffled piston in an open domain; ii) reflection of an acoustic pulse on an oblique wall; iii) a wave generated by a baffled piston and reflected from an oblique wall.

\section{A. Radiation from a baffled piston}

The physical problem is to find the sound field generated by a piston that is baffled. The problem is solved in 2D. This problem is chosen to evaluate the field equation solver in the absence of the rigid wall.

The linearized Euler equations are used:

$$
\begin{aligned}
& \frac{\partial p}{\partial t}+\nabla \cdot \vec{u}=0 \\
& \frac{\partial \vec{u}}{\partial t}+\nabla p=0
\end{aligned}
$$

The initial conditions are:

$$
\begin{aligned}
& u(x, y)=0 . \\
& v(x, y)=0 . \\
& p(x, y)=0 .
\end{aligned}
$$

The challenge of this problem is to solve the discontinuity at time $t$ and at the points $y=c t$, when $c t \leq y_{\max }$, where $c$ is speed of sound. In this test case, the variables are non-dimensionalized. Hence, $c=1$, and the point of discontinuity will be at $y=t$, for $t<y_{\max }$.

The numerical computation was performed over the domain $(x, y) \in[-6,6] \times[0,16]$. For this problem, two boundary conditions are used. The boundary conditions on the wall with piston is

$$
v(x, 0, t)= \begin{cases}V_{0} \cos (\omega t) & (x, 0) \in \text { piston } \\ 0 & \text { otherwise }\end{cases}
$$

where $\omega=4$ is the frequency of the piston, and $V_{0}=1$ is the amplitude. The outflow boundary condition is based on the acoustic radiation condition of Tam and $\mathrm{Web}^{19}$ :

$$
\begin{aligned}
& \frac{\partial \vec{u}}{\partial t}+\nabla p=0 . \\
& \frac{\partial p}{\partial t}+\frac{\partial p}{\partial x} \cos \theta+\frac{\partial p}{\partial y} \sin \theta+\frac{p}{2 r}=0 .
\end{aligned}
$$

where $\theta$ is the angular coordinate of the boundary point, and $r$ is distance from the origin (center of the piston) to the boundary point.

An analytical solution exists for this problem ${ }^{21,22}$ : 


$$
p(x, y, t)=\frac{\rho_{0}}{2 \pi} \int_{s} \int_{-\infty}^{\infty} \frac{\dot{u}\left(x^{\prime}, 0, t-\frac{\sqrt{\gamma^{2}+\varphi^{2}}}{c}\right)}{\sqrt{\gamma^{2}+\varphi^{2}}} d \varphi d x^{\prime}
$$

where the $x^{\prime}$ values are points on the source and $\gamma^{2}=\left(x-x^{\prime}\right)^{2}+y^{2}$, and $\dot{u}_{p}$ is orthogonal speed.

The computation is done on a uniformly spaced grid with $\Delta y=\Delta x=1 / 15$ and a time step $\Delta$ t determined using $\mathrm{CFL}=0.2$, where $\mathrm{CFL}=c \frac{\Delta t}{\Delta x}$. Figure 6 compares the solution given by the OPC-fv scheme with analytical solution demonstrating that there is good agreement between them.

Figure 7 shows contour plots of pressure at $t=8.5$ and $t=20$. The starting pressure is the initial piston face pressure. The piston radiation starts as a collimated plane-wave beam that moves from the face to $\mathrm{r} \cong$ radius of piston, beyond which the beam propagates spherically. This behavior is in concordance with Blackstock's study ${ }^{20}$.

\section{B. Reflection of a Pulse by an Oblique Wall}

A simple example that enables us to check the performance of the cut-cell approach is to solve for the reflected sound from an oblique wall. Even though this problem can be solved by placing the wall parallel to the grid line, we purposely arrange the wall so it is at an oblique angle to the grid line. This offers a direct evaluation of the cut-cell technique. (29).

In this example, the sound hits the wall and reflects. The problem is characterized by the linearized Euler Eq.

The initial condition is

$$
p(x, y)=\exp \left\{-\ln (2)\left[\left(\frac{x-x_{0}}{b}\right)^{2}+\left(\frac{y-y_{0}}{b}\right)^{2}\right]\right\}
$$

where $b=1 . / 6$. The values $x_{0}$ and $y_{0}$ are chosen so that the distance from the point $\left(x_{0}, y_{0}\right)$ to the oblique solid wall is equal to 1.5 .

The outflow boundary condition is the same as that used in the previous test problem. The solid wall boundary conditions for pressure and velocity are given in Eq.(28).

The computation is done on a Cartesian grid that is characterized by $\Delta x=\Delta y=0.05$ and $\mathrm{CFL}=0.5$.

Figure 9 illustrates the solid boundary intersecting a Cartesian mesh. The boundary cell can remain independent, like in $A B C K$, or it can merge with a neighboring cell. For example, $C I D$ merges with $K C I J$. The computation of the parameter in the cut cell $C D I J K$ can be written for pressure from wave equation (29) in the form:

$$
\frac{\partial \bar{p}}{\partial t}+\frac{1}{S_{C D I J K}}\left(u_{C D} d y_{C D}+u_{J K} d y_{J K}-v_{D I} d x_{D I}-v_{I J} d x_{I J}-v_{C K} d x_{C K}-v_{C D} d x_{C D}\right)=0
$$

where the values of $u$ and $v$ represent the values of the function in the middle of the segment. The value of the parameter is approximated using a) the OPC scheme on faces $I J$, $J K$ and $K C$, ii) fourth order polynomial on faces $D I$, and iii) Eq.(28) on face $D F$ (boundary).

To evaluate the performance of the cut cell approach, Figure 10 shows the pressure history from time $=0-4$ and for three wall angles: $90^{\circ}, 81^{\circ}$, and $63^{\circ}$. As apparent from the figure, there is a good agreement between these solutions. Figure 11 shows the pressure contours at three time instants by placing the solid wall at $63^{0}$ inclinations.

\section{Wave generated by a baffled piston and reflected by an oblique wall}

This problem is based on the combined characteristics of the previous two cases. The wave generated by a piston is reflected by an oblique wall. The linearized Euler equation (Eq. (29)) is used in this test case, and the initial condition is the same as that of the baffled piston, Eq. (30).

The rectangular domain over which we do the computation is similar to the previous case (see Figure 12) with: $(\mathrm{x}, \mathrm{y}) \in[-6,6] \mathrm{x}[0,15]$. The bottom of the domain is a piston mounted on a plane rigid baffle, hence the boundary conditions are given by Eq.(31). The velocity and pressure on the solid and open boundaries are identical to those used in the previous case. 
The piston presents the same characteristics as the piston from previous case: $V_{0}=1$ and $\omega=4$. The solution is obtained using the uniform grid $\Delta x=\Delta y=0.05$, and with a time step of $C F L=0.5$. Figure 13 highlights the pressure contours at different time instants.

\section{Conclusion}

A method based on a high order, finite-volume scheme aimed at optimizing the dispersion and dissipation properties and the cut-cell technique aimed at handling geometric variations is presented. The approach is motivated by the need for handling acoustic problems with nonlinearities (using finite volume technique) and a complex geometry (using the cut-cell technique). Selected test cases have been used to demonstrate the performance of the method. The present approach can offer accurate and effective treatment to some important and challenging aspects of acoustic problems.

\section{Acknowledgments}

The present effort has been sponsored by the NASA Constellation University Institute Program (CUIP), Ms. Claudia Meyer program monitor. We appreciate the useful suggestions made by Professors Louis Cattafesta and Mark Sheplak, University of Florida, regarding the test problems. We have also benefited from discussions with Professor Xin Zhang, University of Southampton.

\section{References}

${ }^{1}$ Hardin, J., and Hussaini, M.Y., Computational Aeroacoustics, Springer-Verlag, New York /Berlin, 1992

${ }^{2}$ Tam, C.K.W., "Dispersion-relation-preserving finite difference schemes for computational acoustic", J.Comput. Phys., Vol. 107, No. 2, 1993, pp. 262-281

${ }^{3}$ Tam, C.K.W., Webb, J.C., and Dong, Z., “A study of the Short Wave Components in Computational Acoustics”, J. Comput. Acoust. , Vol. 1, 1993, pp. 1-30

${ }^{4}$ Delfs, J.W., "An overlapping grid Technique for High resolution CAA schemes for Complex geometries", AIAA Paper 20012199, 2001

${ }^{5}$ Shyy, W., Francois, M., Udaykumar, H.S., N'Dri, N, and Tran-Son-Tay, R., "Moving boundaries in micro-scale biofluid dynamics", Applied Mechanics Reviews, Vol. 54, 2001, pp. 405-453

${ }^{6}$ Henshaw, B., “A high-Order Accurate Solver for Maxwell's Equations on Overlaping Grids”, $7^{\text {th }}$ Symposium on Overset Composite Grids and Solution Technology, Oct. 2004

${ }^{7}$ Sherer, S., "High-Order Overset- Grid Acitivities in AFRL/VAAC", Air Forces Research Laboratory, $7^{\text {th }}$ Symposium on Overset Composite Grids and Solution Technology, Oct. 2004

${ }^{8}$ Dumbser, M., “ADER Discontinuous Galerkin Schemes for Aeroacoustics”, CAA: from acoustic sources modeling to far-field radiated noise prediction, EUROMECH Colloquim no. 449, Dec. 2003

${ }^{9}$ Basel, B., and Grünewald, M., "High order unstructured finite difference method in aeroacoustic", CAA: from acoustic sources modeling to far-field radiated noise prediction, EUROMECH Colloquim no. 449, Dec. 2003

${ }^{10}$ Yang, G., and Ingram, D.M., "Cartesian Cut-Cell Method for Axisymmetric Separating Body Flows” AIAA J., Vol. 37, No. 8, 1999, pp. 905-911

${ }^{11}$ Calhoun, D., and Le Veque, R.J., "A Cartesian Grid Finite-Volume Method for the Advection-Diffusion Equation in Irregular Geometries", J. Comput. Phys., Vol.157, 2000, pp. 143-180

${ }^{12}$ Ingram, D.M., Causon, D.M., and Mingham, C.G." Development in Cartesian cut cell methods", Mathematics and Comput. In Simulation, Vol. 61, 2003, pp. 561-572

${ }^{13}$ Udaykumar, H.S., Mittal, R., and Shyy, W., "Computational of Solid-Liquid Phase Fronts in the Sharp Interface Limit on Fixed Grids", J. Comput. Phys., Vol. 153, 1999, pp. 535-574

${ }^{14}$ Ye, T., Mittal, R., Udaykumar, H.S., and Shyy, W., “An Accurate Cartesian Grid Method for Viscous Incompressible Flows with Complex Immersed Boundaries", J. Comput. Phys. , Vol. 156, 1999, pp. 209-240

${ }^{15}$ Ye, T., "Direct numerical simulation of a translating vapor bubble with phase change", Ph.D. Dissertation, University of Florida, Department of Mechanical Engineering, Gainesville FL, 2001

${ }^{16}$ Ashcroft, G., and Zhang, X., “Optimized Prefactored Compact Scheme”, J. Comput. Phys., Vol. 190 , 2003, pp. 459-477

${ }^{17}$ Popescu, M., Shyy, W., and Garbey, M., "A Study of Dispersion-Relation-Preserving and Optimized Prefactored Compact Schemes for Wave Equation”, AIAA paper 2004-0519, 2004 , also accepted for publication in J. Comput. Phys.

${ }^{18} \mathrm{Hu}$, F.Q., Hussaini, M.Y., and Manthey, J.L., "Low Dissipation and Dispersion Runge-Kutta for Computational Acoustics", $J$. Comput. Phys., Vol. 124, 1996, pp. 177-191 
${ }^{19}$ Tam, C.K.W., and Webb, J.C,. "Radiation Boundary Condition and Anisotropy Correction for Finite Difference Solutions of the Helmholtz Equation”, J. Comput. Phys., Vol. 113, 1994, pp. 122-133

${ }^{20}$ Blackstock, D.T., Fundamental Of Physical Acoustic, Wiley, New York, 2000

${ }^{21}$ Williams, E.G., Fourier Acoustics Sound Radiation and Nearfield Acoustical Holography, Academic Press, Dan Diego, 1999

${ }^{22}$ Morse, P.M., and Incard, K.U., Theoretical Acoustic, McGraw-Hill Book Company, New York, 1968

${ }^{23}$ Kim,. J.W., and Lee, D.J., "Optimized Compact Finite Difference Schemes with Maximum Resolution”, AIAA J., Vol. 34, No. 5, 1996, pp. 887-893

${ }^{24}$ Tam, C.K.W., and Kurbatskii, K.A., "A Wavenumber Based Extrapolation and Interpolation Method for Use in Conjunction with High-Order Finite Difference Schemes”, J. Comput. Phys., Vol. 157, 2000, pp. 588-617 


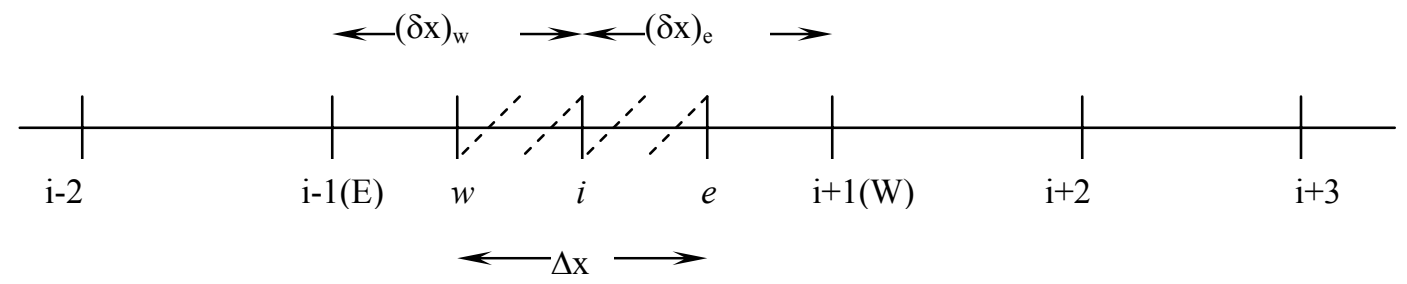

Figure 1. Grid points cluster for one-dimensional problem

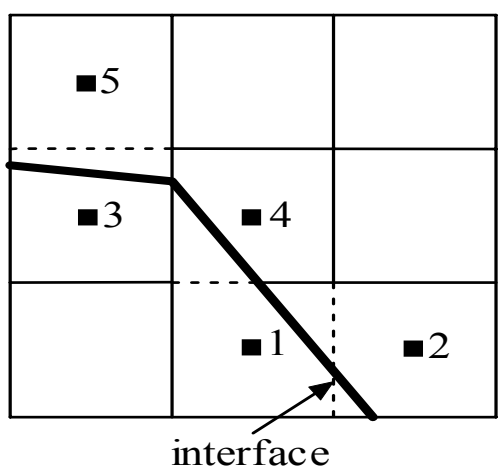

a)

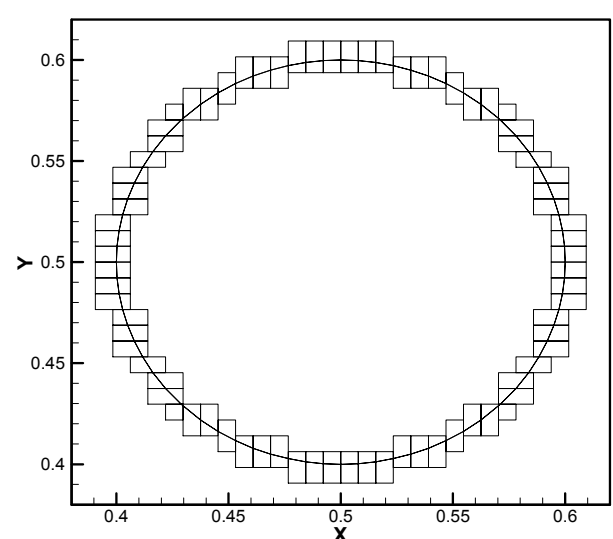

b)

Figure 2. Illustration of the interfacial cells and cut-and-absorption procedures: a) local situation, b) cut-cells along the interface.

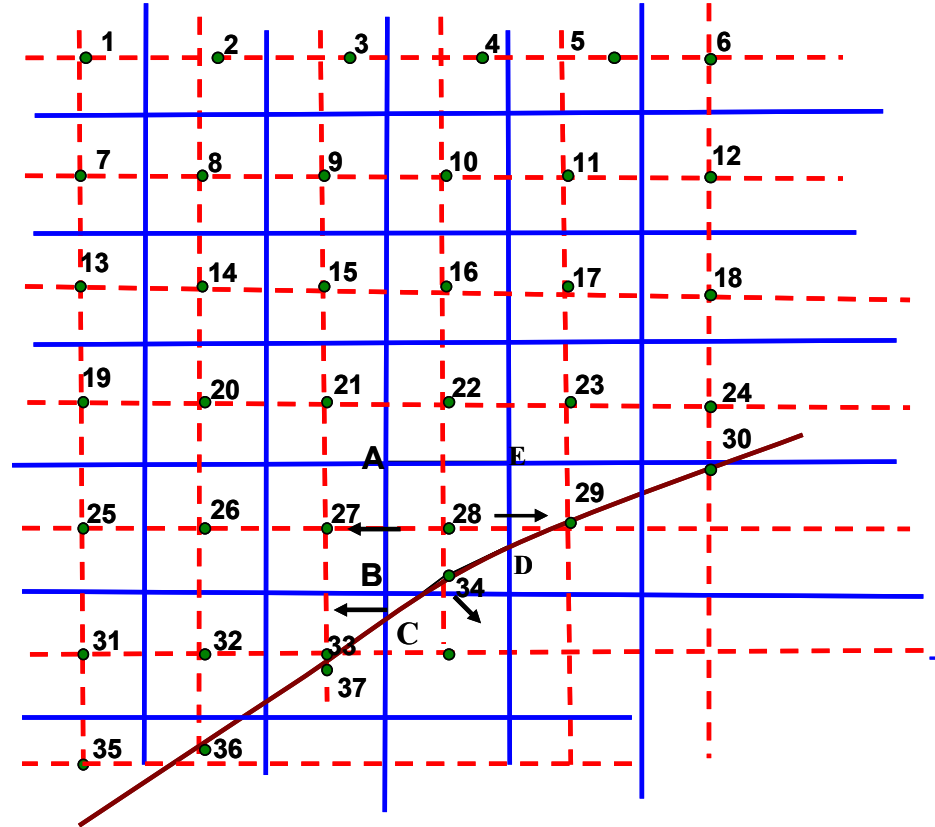

a)

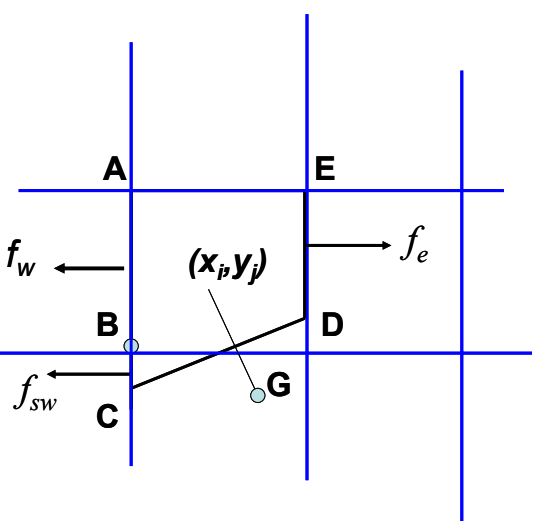

b)

Figure 3. Modified cut - cell approach for CAA: a)Cartesian cut cell approach; b) Detail around of the cut cell 


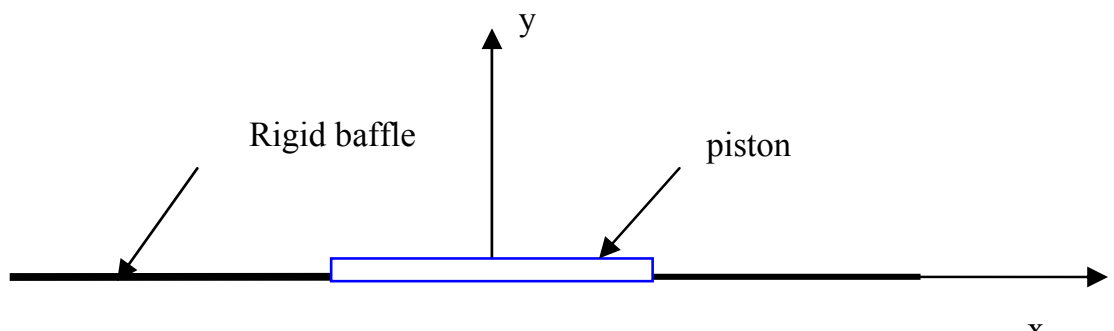

Figure 4. Radiation from a baffled piston (test problem (ii)): general description

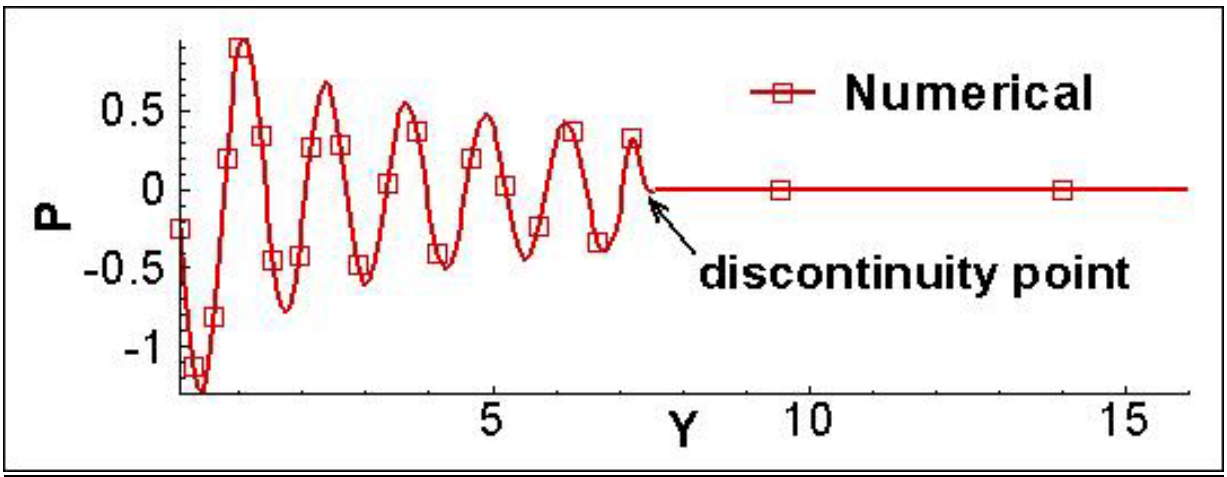

Figure 5. Radiation from a baffled piston: Numerical solution at $t=7.3<Z_{\max }$

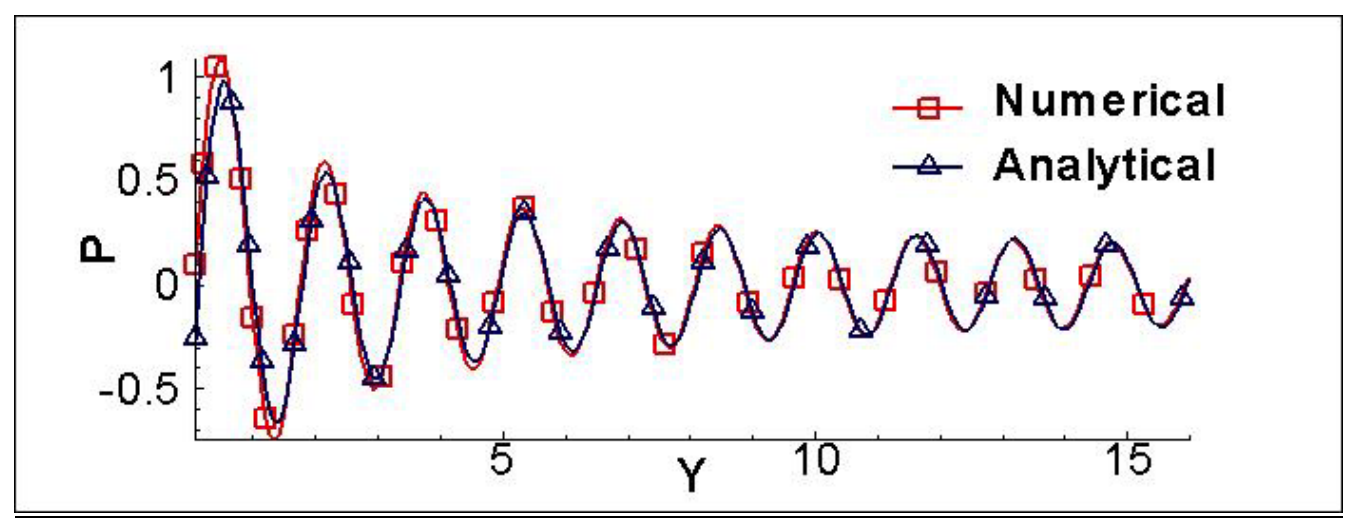

Figure 6. Radiation from a baffled piston: Comparison between analytical and computed solutions on axis 


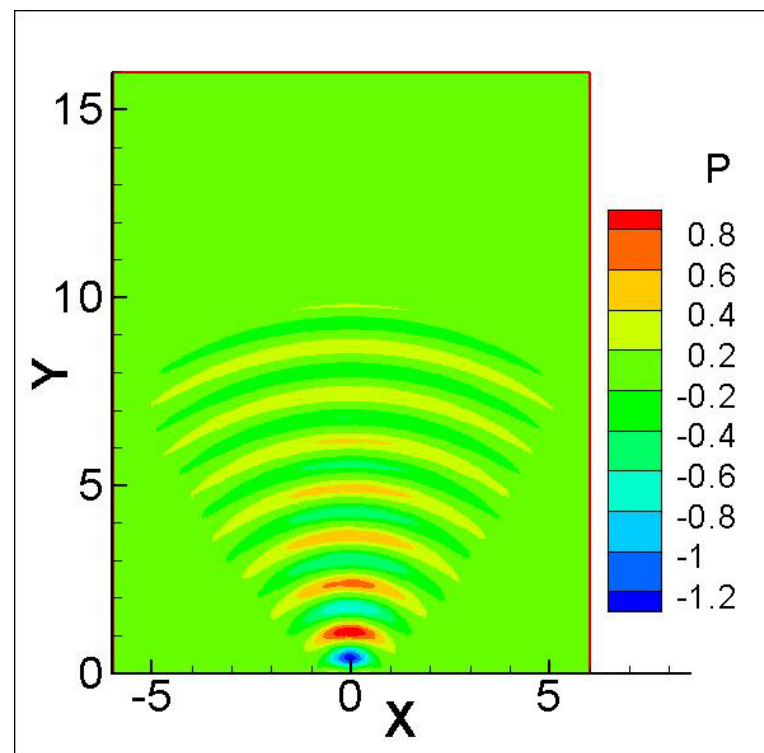

a)

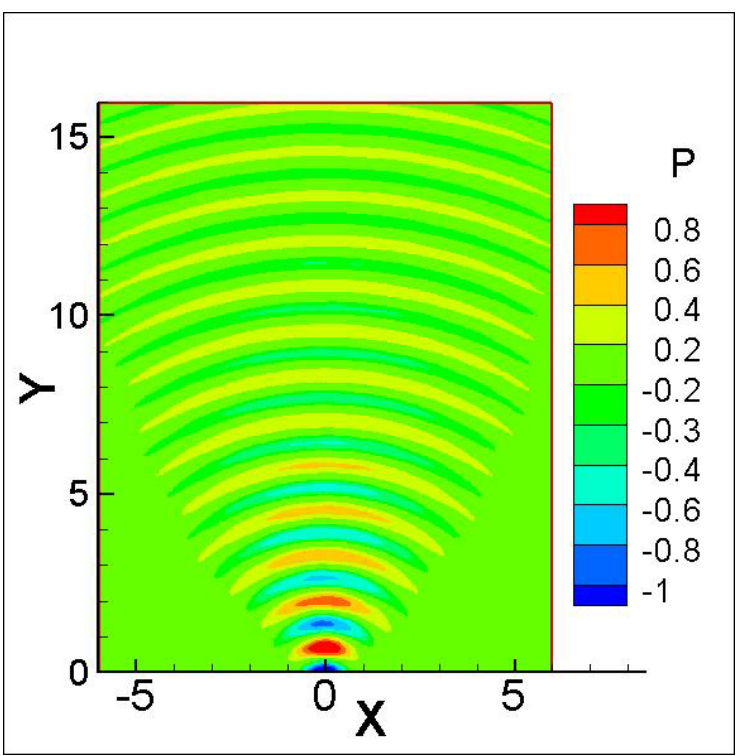

b)

Figure 7. Radiation from a baffled piston: Contour plot of the pressure-numerical solution at: a) $t=8.5$; b) $t=20.0$

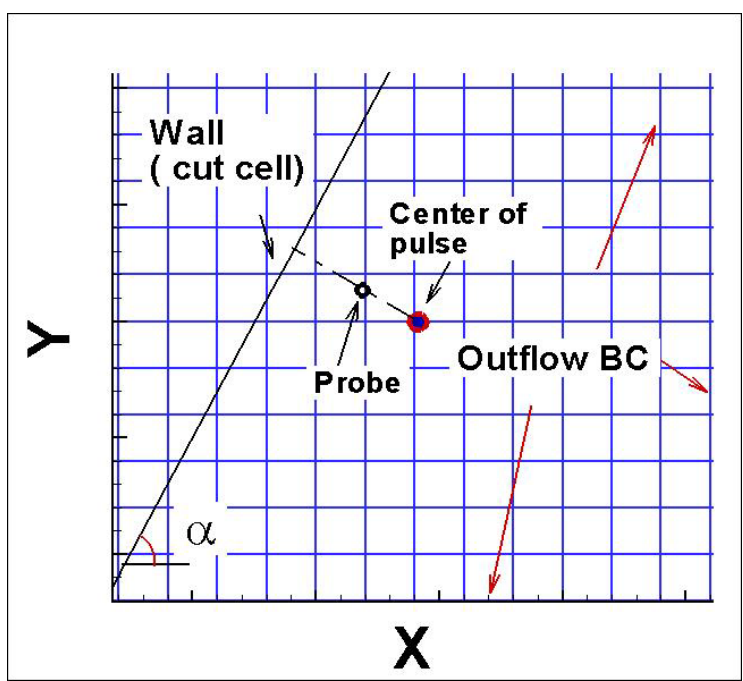

Figure 8. Reflection of the pulse by an oblique wall (test problem (ii)): general description 


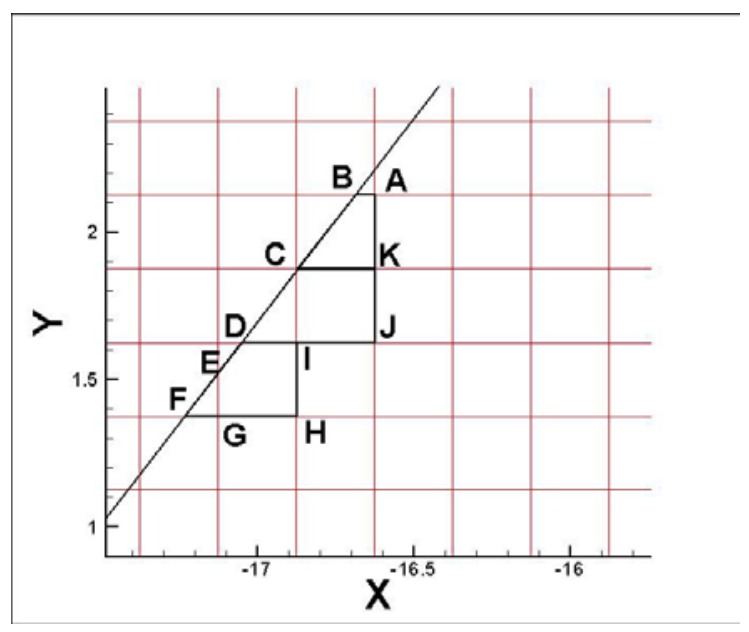

Figure 9. Reflection of the pulse by an oblique wall (test problem (ii)): cell around boundary

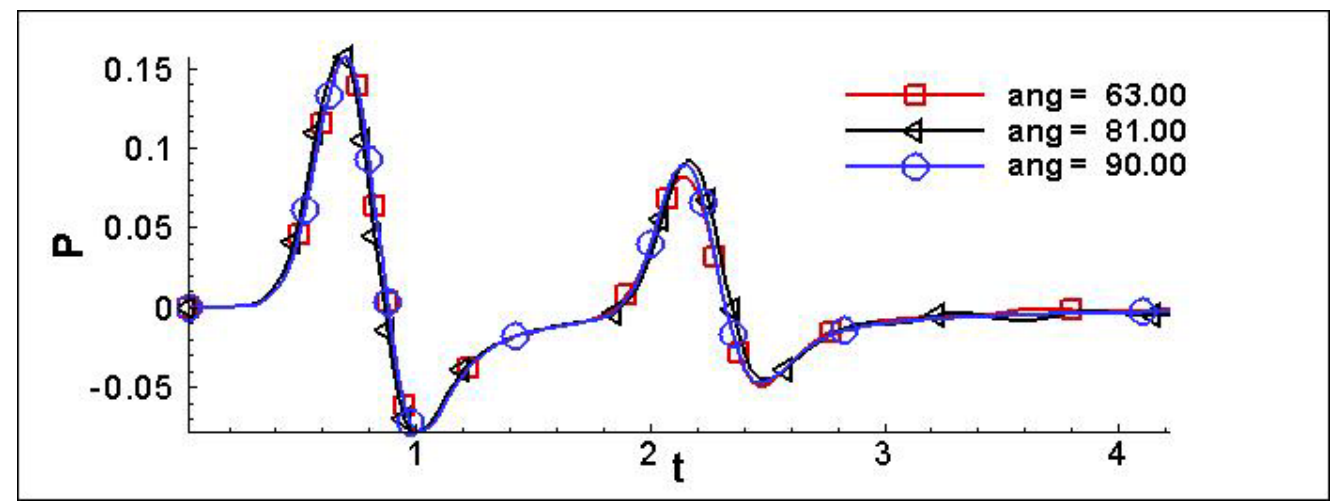

Figure 10. Reflection of the pulse by an oblique wall: history of pressure for different angle of wall

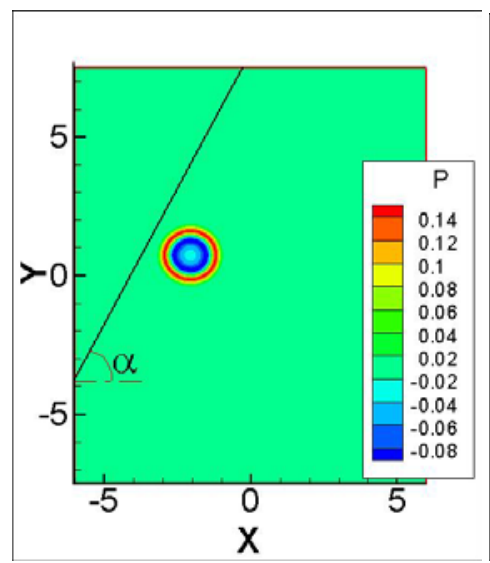

a)

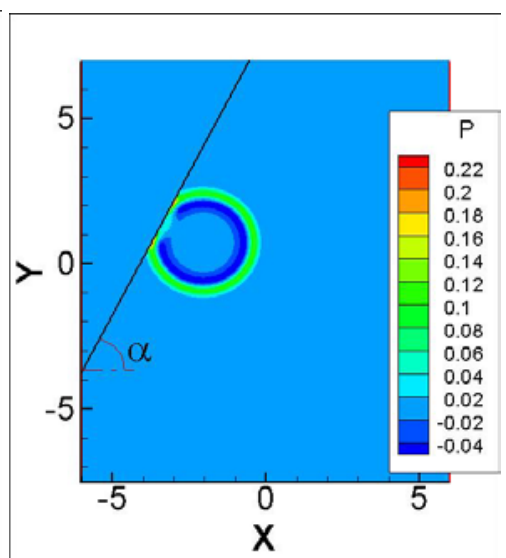

b)

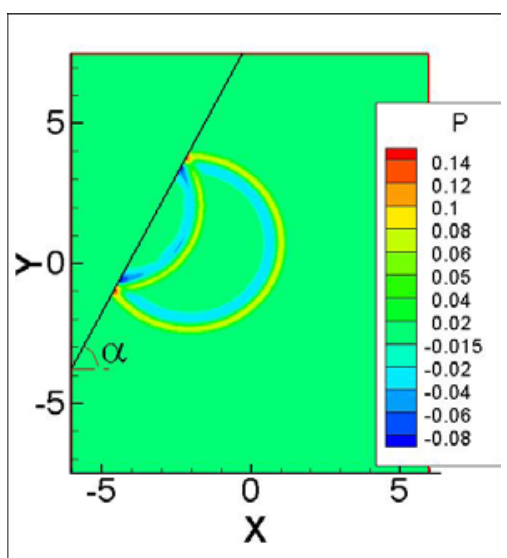

c)

Figure 11. Reflection of the pulse by an oblique wall: $\alpha=63^{0}$ a) $t=0.8$; b) $t=1.6$; c) $t=3.2$; 


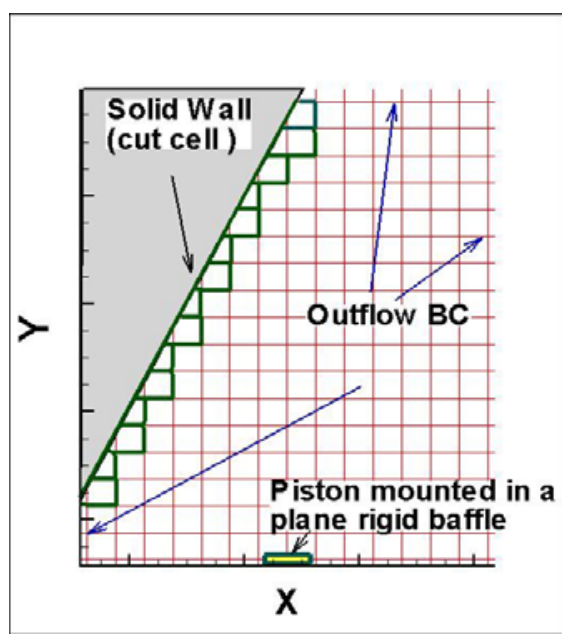

Figure 12. Wave generated by a baffled piston and reflects an oblique wall (test problem (iii)): General description of the domain

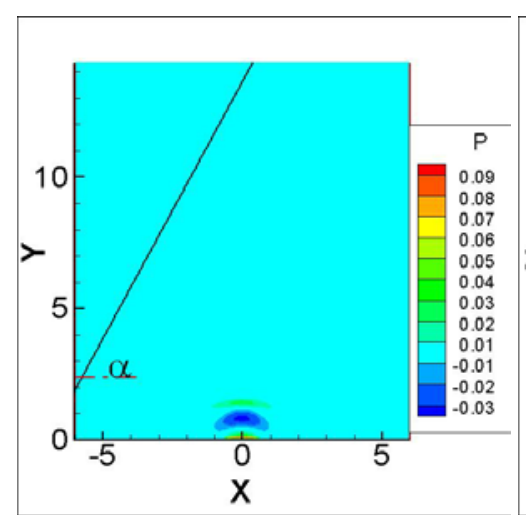

a)

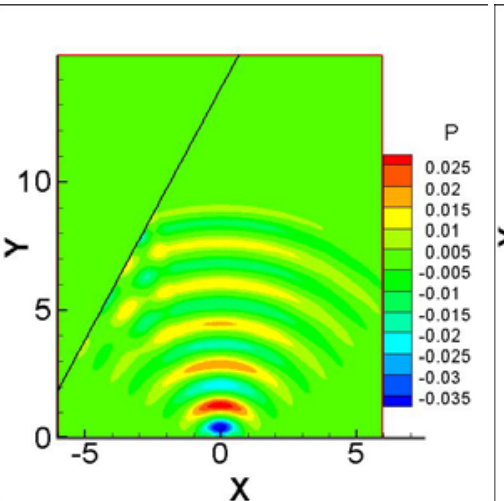

b)

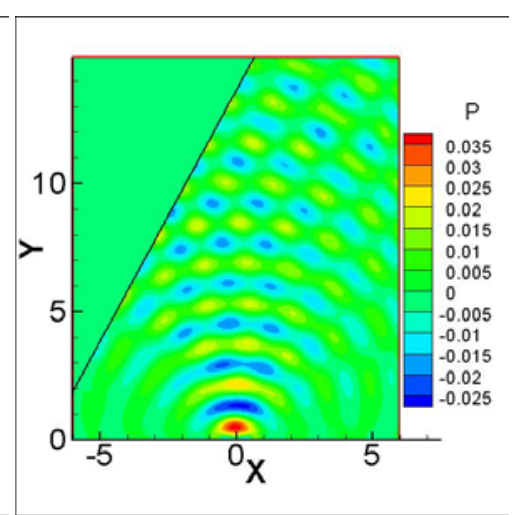

c)

Figure 13. Wave generated by a baffled piston and reflects an oblique wall: $\alpha=63^{0}$; a) $t=1.5$; b) $t=9.4$; c) $t=25.0$ 